\title{
Volume Relaxation in Amorphous Polymers around Glass-Transition Temperature
}

\author{
Masataka UchIDoI, Keiichiro ADACHI, and Yoichi IsHIDA \\ Department of Polymer Science, Faculty of Science, \\ Osaka University, Toyonaka, Osaka, 560 Japan.
}

(Received June 15, 1977)

\begin{abstract}
Relaxation of volume, enthalpy, and dielectric polarization have been studied for poly(vinyl acetate) and polystyrene around a glass-transition point. It has been concluded that the correlation time of microbrownian motion in glassy polymers shifts with time during the period of volume relaxation. The time dependence of dielectric relaxation time $\tau_{\mathrm{D}}$ has been represented approximately by
\end{abstract}

$$
\log \tau_{\mathrm{D}}=a \log t-b
$$

where $a$ and $b$ denote constants. By application of this equation to the volume and enthalpy relaxation processes, the time dependence of the specific volume $V(t)$ or enthalpy $H(t)$ can be represented in the form

$$
V(t)-V(\infty)=c t^{-B}
$$

where $B$ and $c$ are constants. The values of $B$ for the volume relaxation agree well with those for the enthalpy.

KEY WORDS: Volume Relaxation/Dielectric Relaxation/Enthalpy Relaxation / Poly(vinyl acetate) / Polystyrene / Glassy State /

The glass-transition temperature $T_{\mathrm{g}}$ of amorphous polymer is the temperature at which the correlation time of microbrownian motion is measured in experimental scale. Below $T_{\mathrm{g}}$, the internal state is usually not in equilibrium on account of the prolonged relaxation time. ${ }^{1,2}$ If a polymer is allowed to stand long enough even at a temperature below $T_{\mathrm{g}}$, its internal state will approach equilibrium gradually accompanied by variation of thermodynamic quantities such as volume, enthalpy, etc. Study of volume relaxation is essential for understanding the glass transition and the glassy state of amorphous polymer.

Volume relaxations of polystyrene and poly(vinyl acetate) were studied by Kovacs, who showed the volume relaxation curves measured at different temperatures are superposable. ${ }^{3} \mathrm{He}$ explained the shape of the curves assuming that the relaxation time depends not only on temperature but also on free volume fraction. ${ }^{4}$ Goldstein and Nakonocznyj studied volume relaxation on zinc chloride and tried to explain the data in terms of the wide distribution of relaxation time. ${ }^{5}$ Hozumi et al., also measured volume relaxation of poly- styrene $^{6}$ and analyzed the results in terms of Bueche's theory. ${ }^{7}$ However these authors could not satisfactorily explain the difference among relaxation curves measured after being subjected to different thermal histories.

The correlation time for microbrownian motion has been generally related to free volume as proposed by Doolittle. ${ }^{8}$ On the other hand, Adam and Gibbs expressed the correlation time as a function of configurational entropy and temperature. ${ }^{9}$ From these theories, we can infer that the correlation time depends not only on temperature but also on the internal state. The relaxation time in the volume relaxation process would vary with specific volume due to feedback effect as indicated by Kovacs. ${ }^{4}$

The purpose of the present study is to clarify how the relaxation time depends on time and also to formulate the volume relaxation curve as a function of time. Also the relaxations of different thermodynamic quantities are compared. Thus the volume and enthalpy relaxations have been measured on poly(vinyl acetate) and polystyrene. In order to investigate the time dependence of 
correlation time of microbrownian motion, we measured the shift of dielectric relaxation time in the course of the volume relaxation.

A similar dielectric study just below glass transition point has already been made by Kästner who showed that the dielectric loss factor corresponding the tail of the loss peak decreased gradually with volume relaxation. ${ }^{10}$ However, he could not observe a shift in the loss peak since he measured at a relatively high-frequency range. We cannot judge from his data whether the loss peak actually shifted to a lower frequency or the magnitude of dispersion decreased with the volume relaxation as claimed by Williams ${ }^{11}$ and Saito and Nakajima. ${ }^{12}$ In this study, we have carried out dielectric measurements in a low-frequency range where we can observe the loss peak. A study of the mechanical relaxation in the course of volume relaxation was reported by Kovacs, et al. ${ }^{13}$ They showed that $\tan \delta$ for shear deformation varied with time below the glass-transition point. However, they did not explicitly show the time dependence of the relaxation time.

\section{EXPERIMENTAL}

\section{Samples}

Poly(vinyl acetate) (PVAC) waspr epared by radical polymerization in methanol at $60^{\circ} \mathrm{C}$ using azobisisobutylonitrile as the initiator. The polymer was recovered from the reaction mixture by precipitating in an excess of petroleum ether under vigorous stirring and was dried under a vacuum of $10^{-2}$ torr for several days. Polystyrene (PS) was a commercial sample (Dow Chemical Co.) purified by precipitating in methanol from benzene solution. The viscosity-average molecular weights of the PVAC and the PS samples were $2.5 \times 10^{5}$ and $1.0 \times 10^{5}$, respectively. The glasstransition temperatures of the PVAC and the PS were $43^{\circ}$ and $103^{\circ} \mathrm{C}$, respectively, determined by a differential scanning calorimetry at the heating rate of $10^{\circ} \mathrm{C} / \mathrm{min}$. Films for dilatometric measurements with a thickness of about $0.5 \mathrm{~mm}$ were obtained by pressing the bulk PVAC and PS samples, respectively, at $140^{\circ}$ and $200^{\circ} \mathrm{C}$ under a vacuum of $10^{-2}$ torr. Bubble-free portions of the films were cut into pieces of $0.5-\mathrm{cm}$ square and used for the measurement.

\section{Dilatometry}

Dilatometer is schematically shown in Figure 1. The area of the cross section of capillary was $2.70 \pm 0.05 \times 10^{-3} \mathrm{~cm}^{2}$. The amount of the PVAC and the PS sealed in dilatometers were 2.779 and $6.070 \mathrm{~g}$, respectively. After the specimens were sealed in a dilatometer, they were degassed under a vacuum of $10^{-5}$ torr for ten hours at $120^{\circ} \mathrm{C}$ for the PVAC and $220^{\circ} \mathrm{C}$ for the PS. The fluctuation of temperature in a bath was regulated within $\pm 0.1^{\circ} \mathrm{C}$. The specific volume was measured pycnometrically at $27^{\circ} \mathrm{C}$.

\section{Dielectric Measurement}

Dielectric measurements in the ultra lowfrequency region were performed by means of the transient current method using an electrometer model 640 (Keithley, Ohio). Transient currents measured from 2 to $3 \times 10^{5} \mathrm{sec}$ have been transformed into the cmplex dielectric constant $\varepsilon^{*}$ by

$$
\varepsilon^{*}-\varepsilon_{\infty}=\frac{1}{C_{0} E} \int_{0}^{\infty} I(t) \mathrm{e}^{i \omega t} d t
$$

where $\omega, C_{0}, E$, and $I(t)$ denote angular frequency, the capacitance of the empty condenser, applied voltage, and transient current respectively. In this transformation, $I(t)$ in the region of less than $2 \mathrm{sec}$ and that longer than $3 \times 10^{5} \mathrm{sec}$ have been estimated by exponential extrapolation. The calculation was performed with an electronic computer. Since a time scale of $t \mathrm{sec}$ corresponds

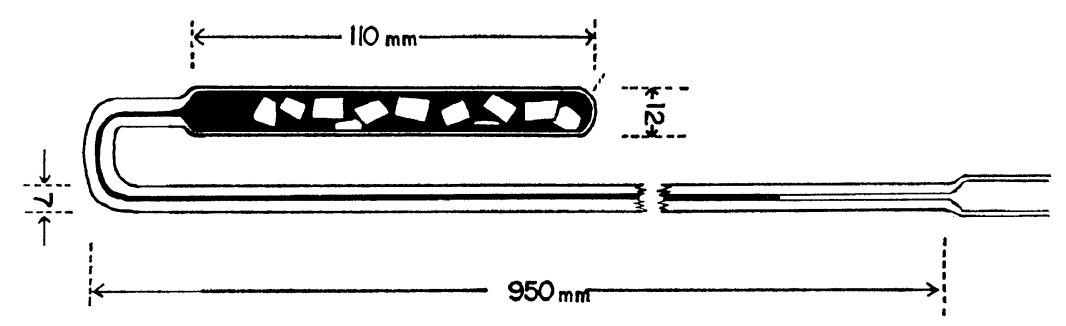

Figure 1. Cross sectional view of a dilatometer. 
to the frequency scale of $(2 \pi t)^{-1}, \varepsilon^{\prime \prime}$ thus calculated is reliable at least in the range of $\log f$ from -1.1 to -6.3 .

\section{Enthalpy Measurement}

The enthalpy relaxations were measured on the PVAC by means of a Calvet microcalorimeter (Setram, Lyon). A glass ampule containing $1.987 \mathrm{~g}$ of the PVAC was used for the measurement.

\section{Procedure of Temperature Jump}

In order to observe the volume or enthalpy relaxations around $T_{\mathrm{g}}$, we employed a temperature jump technique, in which the sample temperature was changed rapidly from an initial temperature $T_{1}$ to an annealing temperature $T_{\mathrm{a}}$. The temperature jump should be accomplished instantaneously. In volume measurements, a dilatometer was initially immersed in a bath of temperature $T_{\mathrm{i}}$, and subsequently transferred to another bath of temperature $T_{\mathrm{a}}$. The temperature jump was also performed by changing the temperature of the bath rapidly. In either case the temperature equilibrium was attained in three minutes. Enthalpy measurements were carried out as follows. An ampule containing the PVAC was heated up to $100^{\circ} \mathrm{C}$, and then immersed for five minutes in a water bath of temperature $T_{\mathrm{a}}$, and then set in the calorimeter. It took $30 \mathrm{~min}$ to attain an equilibrium temperature. In dielectric measurements, it also took $30 \mathrm{~min}$ to attain equilibrium.

\section{RESULTS AND DISCUSSION}

\section{Volume Relaxation}

Figure 2 shows examples of the volume relaxation curves for the PVAC after respective temperature jumps described in the figure. $V$ and $\log t$ denote the specific volume and the common logarithm of the elapsed time subsequent to the temperature jump. The shapes of volume relaxation curves subsequent to the temperature jump from $55^{\circ} \mathrm{C}$ to a given temperature $T_{\mathrm{a}}$ agreed with those curves from $100^{\circ} \mathrm{C}$ to the corresponding $T_{\mathrm{a}}$. Therefore, we can conclude that the initial temperature $T_{1}$ does not affect the observed behavior of volume relaxation if $T_{\mathrm{i}}$ is higher than $T_{\mathrm{g}}+10^{\circ} \mathrm{C}$. As is seen in the figure, the total variation of volume during annealing was increased with a decrease of $T_{\mathrm{a}}$. The logarithm of the slope of the

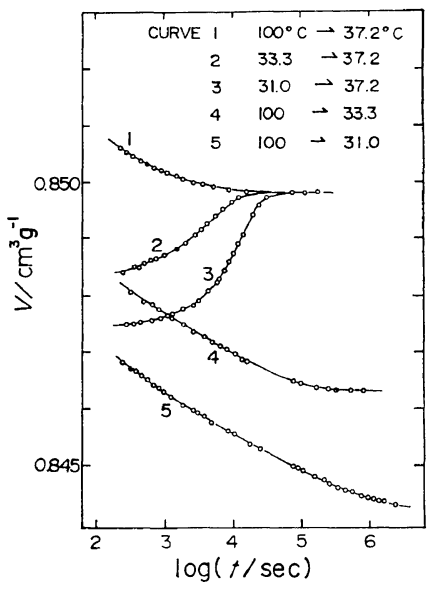

Figure 2. Volume relaxations of the PVAC at various temperatures, $T_{\mathrm{a}}$ after being subjected to various modes of temperature jumps as indicated.

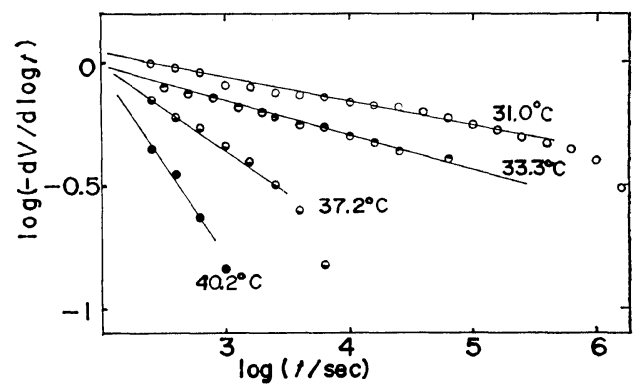

Figure 3. Time dependence of the slopes of volume relaxation curves of the PVAC.

curves $\log (-\mathrm{d} V / \mathrm{d} \log t)$ varies in an approximately linear manner with $\log t$ as shown in Figure 3. The dependence of $\log (-\mathrm{d} V / \mathrm{d} \log t)$ on temperature becomes smaller with a decrease in the annealing temperatures. This means that the volume varies in proportion to $\log t$ in a region relatively far below $T_{\mathrm{g}}$. This linearity was already pointed out by Fox and Flory for polystyrene. ${ }^{14}$ It should be noted that in the period in which the relaxation terminates, $\log (-\mathrm{d} V / \mathrm{d} \log t)$ decreases rapidly, as can be seen in Figure 3.

The curves 2 and 3 in Figure 2 are examples of the volume relaxation subsequent to an upward temperature jump, i.e., from $T_{1}$ which is lower than $T_{\mathrm{a}}$. The curve 2 was observed when the sample was annealed at $33.3^{\circ} \mathrm{C}$ for 3.5 days to attain equilibrium (compare with curve 4) and subsequently heated up to $37.2^{\circ} \mathrm{C}$. The curve 3 was obtained similarly. In these curves (curves 2 


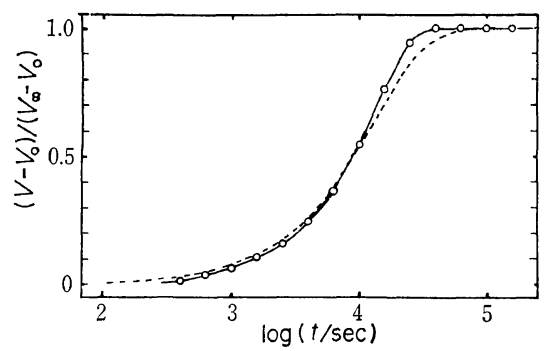

Figure 4. Comparison between the observed volume variation and the single relaxation process. Key $O$ shows observed values of the PVAC in the case where $T_{\mathrm{i}}$ and $T_{\mathrm{a}}$ are $31.0^{\circ}$ and $37.2^{\circ} \mathrm{C}$ respectively. The dotted line shows sigmoidal curve $1-\exp (-t / \tau)$ where $\tau$ is taken to be $12860 \mathrm{sec}$. It should be noted that the dotted line shifts without changing its shape when other value of $\tau$ is used, since $\log t$ is taken for abscissa.

and 3), the value of $\mathrm{d} V / \mathrm{d} \log t$ increases with $\log t$ except for the final stage of relaxation. This contrasts with the behavior of curves 1,4 , and 5 . The shape of curve 3 has been compared with that of a sigmoidal curve with a single relaxation time expressed by $1-\exp (-t / \tau)$. The value of $\tau$ is taken so that the sigmoidal curve agrees with the experiments as closely as possible. As shown in Figure 4, the observed curve of the volume relaxation has a larger slope than the sigmoidal curve. This fact cannot be explained by assuming a distribution of relaxation times, which only broadens the sigmoidal curve. Accordingly we can conclude that the volume relaxation process cannot be accounted for in terms of the distribution of the relaxation times alone. From the comparison of the shapes of the curves 1,2 and 3 it is also concluded that the rate of the relaxation varies with the specific volume, although the temperature is the same. These facts strongly suggest that the relaxation time shifts with the lapse of time after temperature jump.

\section{Enthalpy Relaxation}

Enthalpy relaxation was measured at $31^{\circ}$ and $35^{\circ} \mathrm{C}$. The results are shown in Figure 5. Since the reading of the calorimeter is in heat evolution per unit time, the value of $\log (-\mathrm{d} H / \mathrm{d} t)$ is plotted for the ordinate. As shown in the figure, log $(-\mathrm{d} H / \mathrm{d} t)$ is linear to $\log t$ having a slope smaller than -1 . This behavior is similar to that of the volume relaxation.

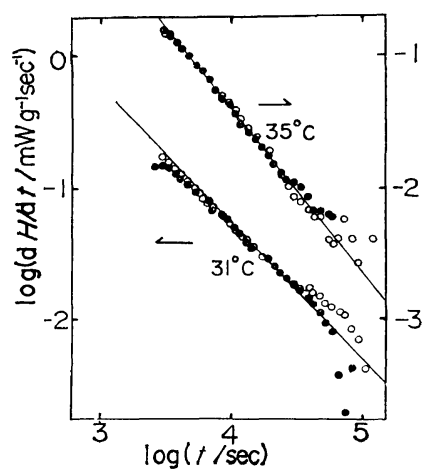

Figure 5. Slopes of enthalpy relaxations of the PVAC at 31.0 and $35.0^{\circ} \mathrm{C}$.

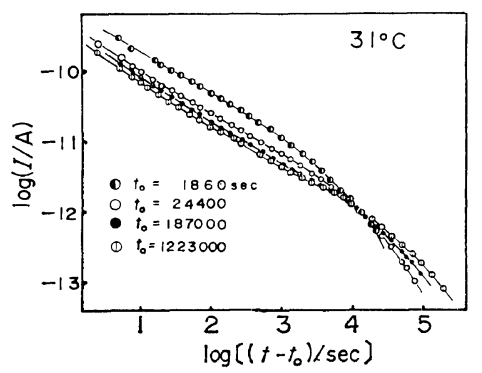

Figure 6. Transient currents of the PVAC. Measurements were started at respective times $\left(t_{0}\right)$ after temperature jump from $55^{\circ}$ to $31^{\circ} \mathrm{C}$. $t$ denotes the elapsed time after the temperature jump.

\section{Dielectric Relaxation}

The transient current after the temperature jump from $55^{\circ} \mathrm{C}$ to $31^{\circ} \mathrm{C}$ is shown for the PVAC in Figure 6. In this figure, the starting time $t_{0}$ represents the time required for annealing prior to the start of current measurements. The curve whose $t_{0}$ is $1860 \mathrm{sec}$ is taken from a charging current measurement. The others are the discharging current measurements. It has been confirmed that the charging-current curve is superposable with the discharging one if the leak current is subtracted from the charging current. As shown in Figure 6, the time scale of the current measurements is $10^{5} \mathrm{sec}$. On the other hand, the volume recovery at $31^{\circ} \mathrm{C}$ completed at $10^{6}$ to $10^{7} \mathrm{sec}$. The dielectric relaxation time is about one tenth of the volume relaxation time. The scale of segmental motion associated with dielectric relaxation process may be much smaller than that for volume. The frequency dependence of the dielectric loss factors $\varepsilon^{\prime \prime}$ of the PVAC transformed 


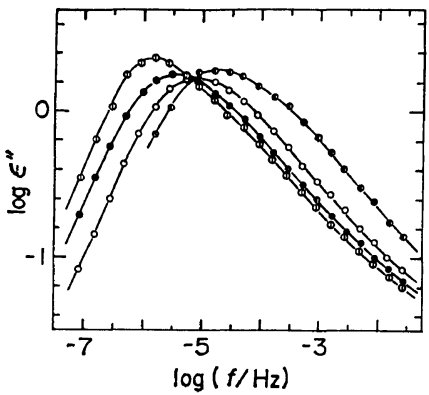

Figure 7. Frequency dependence of dielectric loss factors of the PVAC which were calculated by Fourier transformation of the transient currents is shown in Figure 6: $O, t_{0}=1860 \mathrm{sec} ; O, t_{0}=24400 \mathrm{sec}$; $t_{0}=187000 \mathrm{sec} ;$ (1) $t_{0}=1223000 \mathrm{sec}$.

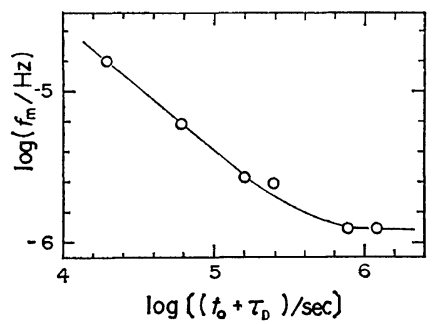

Figure 8. Annealing time dependence of the frequency where loss becomes a maximum.

from the transient currents is shown in Figure 7. As shown in the figure, the frequency $f_{\mathrm{m}}$ at which loss becomes maximum shifts with an increase in starting time $t_{0}$. Since the mean dielectric relaxation time $\tau_{\mathrm{D}}$ is given approximately by

$$
\tau_{\mathrm{D}}=1 /\left(2 \pi f_{\mathrm{m}}\right)
$$

we can conclude that the dielectric relaxation time increases with the increase of annealing time. It should be noted that when the dielectric relaxation time shifts gradually during the currnt measurement, the current is non-linear in the sense that the current does not follow the superposition principle of Boltzmann. In principle, we cannot obtain a true dielectric loss factor by Fourier transformation of the current. However, influence of the shift of the relaxation time on the current may be small since the time scale for the current measurement is much smaller than the volume relaxation time. When the dielectric relaxation time is gradually prolonged in measurement of the current, the curve of the absorption current has an elongated shape in the direction of the time axis, compared with the curve, when there is no shift in the relaxation time. Therefore, the shift of the relaxation time affects the current so that distribution of the relaxation time seems slightly broadened. In fact, the loss peak transformed from the current at about $1860 \mathrm{sec}$ is broader than the loss peak for the current whose $t_{0}$ is $1.223 \times 10^{6} \mathrm{sec}$ according to Figure 7. Deviation of $\log f_{\mathrm{m}}$ from the true value due to the nonlinear effect is estimated to be less than 0.2 for a single relaxation process. Thus $\log f_{\mathrm{m}}$ may be affected slightly by this effect. The shift of log $f_{\mathrm{m}}$ is shown in Figure 8 where we took $t_{0}+\tau_{\mathrm{D}}$ as the mean value of annealing time $t$ because it took about $2 \tau_{\mathrm{D}} \mathrm{sec}$ for the current measurements. As can be seen in the figure, $\log f_{\mathrm{m}}$ decreases linearly with $\log t$ in the time region where the volume is relaxing. Thus the time dependence of $\tau_{\mathbf{D}}$ can be represented approximately in the form

$$
\tau_{\mathrm{D}}=t^{a} / b
$$

where $a$ and $b$ denote constants.

\section{Formulation of the Volume Relaxation}

Now we shall analyse the volume relaxation as a function of annealing time. According to Kovacs, ${ }^{4}$ the rate of variation of the specific volume $V(t)$ at time $t$ may be written as

$$
\mathrm{d} V(t) / \mathrm{d} t=-[V(t)-V(\infty)] / \tau_{\mathrm{v}}(t)
$$

where $\tau_{\mathrm{v}}(t)$ is the relaxation time for the volume change. This equation defines $\tau_{\mathrm{v}}$ as having a similar $t$-dependence to $\tau_{\mathrm{D}}$. We assume that $\tau_{\nabla}$ also shifts analogously to eq 3 in a period before the relaxation-terminates. Thus, the time dependence of $\tau_{\mathrm{v}}$ may take the form

$$
\tau_{\mathrm{v}}=t^{A} / B
$$

where $A$ and $B$ are adjustable parameters. Substitution of eq 5 into eq. 4 gives the following equations for the time dependence of specific volume;

$$
\begin{aligned}
& V(t)-V(\infty)=c \exp \left[-B t^{1-A} /(1-A)\right] \\
& \text { for } A \neq 1
\end{aligned}
$$

and

$$
V(t)-V(\infty)=c t^{-B} \quad \text { for } A=1
$$

where $c$ is a constant. From eq 4 and 6, the rate of the variation of specific volume is expressed by 


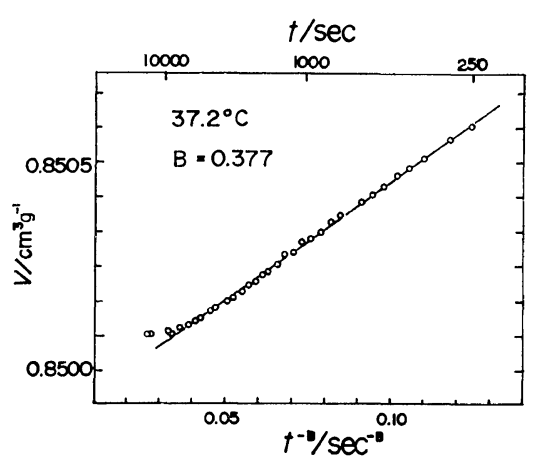

Figure 9. Inspection of linearity of the variation of volume with respect to $t^{-B}$. Key $\bigcirc$ showes the experimental results at $37.2^{\circ} \mathrm{C}$ for the PVAC. The value of $B$ is taken to be 0.377 .

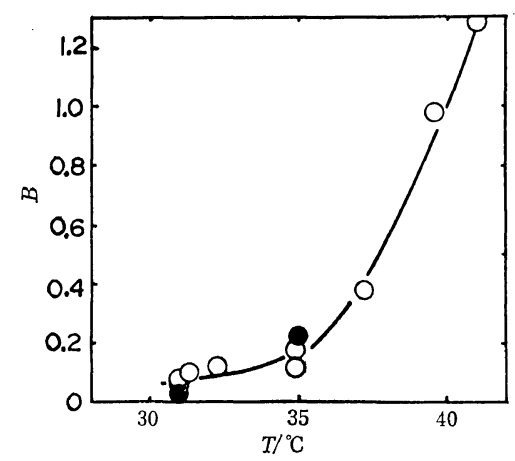

Figure 10. $B$ in eq 7 vs. annealing temperature: $O$, determined from the volume relaxations of the PVAC; 0 , determined from the enthalpy relaxations of the PVAC.

$$
\mathrm{d} \log (-\mathrm{d} V(t) / \mathrm{d} \log t) / \mathrm{d} \log t=1-A-B t^{1-A}
$$

The experimental values of $\mathrm{d} V(t) / \mathrm{d} \log t$ varied linearly with respect to $\log t$ as demonstrated in Figure 3. Therefore, we can take $A=1$, because eq 8 is independent of time. The comparison between eq 7 and the experiment for the PVAC at $37.2^{\circ} \mathrm{C}$ is shown in Figure 9; the parameter $B$ was set as 0.377 . The agreement between eq 7 and the experiment is fairly good except for the final stage of the relaxation. It should be noted that the value of $B$ is independent of the unit in volume measurement. An equation having the form of eq 7 has been also applied to the enthalpy relaxation. The values of $B$ in the volume and enthalpy relaxations of the PVAC determined at various temperatures are shown in Figure 10. Similar analysis has been carried out also for the volume relaxation of the PS. The comparison of eq 7

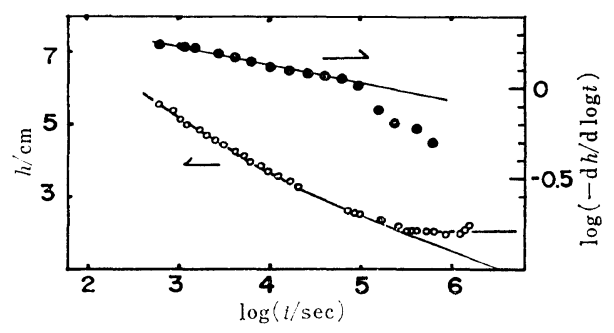

Figure 11. Annealing time dependences of the volume $(\bullet)$ and the slope of volume variation $(\bigcirc)$ for the PS at $94.7^{\circ} \mathrm{C}$. The solid line shows eq 7. Here $h$ denotes the reading of the height of mercury column of the dilatometer.

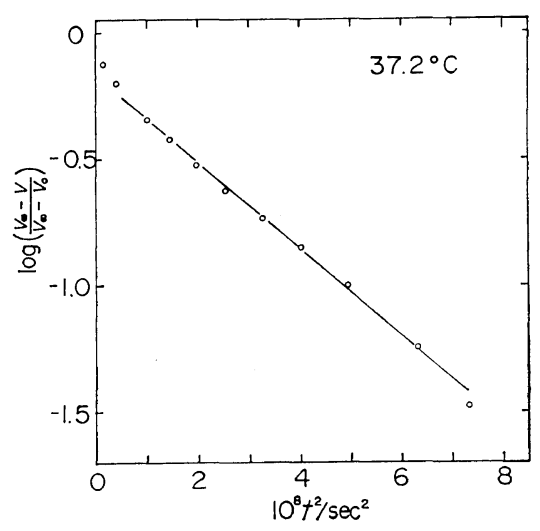

Figure 12. Inspection of linearity of logarithm of volume with respect to $t^{2}$ in eq 10 . Key $\bigcirc$ shows the experimental results given in Figure 4.

with the experiment is shown in Figure 11, where the solid line shows eq 7 .

In the case where $T_{\mathrm{i}}$ is lower than $T_{\mathrm{a}}$, the mean volume relaxation time $\tau_{\nabla}$ decreases with the time, and we may assume the parameter $A$ is negative in eq 5. Assuming $A$ is to be equal to -1 , we can express the time dependence of volume by

$$
V(t)-V(\infty)=c \exp \left[-B t^{2} / 2\right]
$$

The comparison between eq 9 and the experiment at $37.2^{\circ} \mathrm{C}$ is given in Figure 12 . The agreement between them is fairly good except for the initial stage of the relaxation process, as opposed to the case of downward temperature jump in which the agreement was poor at the final stage.

Acknowledgements. The authors are grateful to Prof. Syuzo Seki for his providing the calorimeter. The authors are also indebted to Prof. 
Tadao Kotaka and Dr. Shinsaku Uemura for helpful comments.

\section{REFERENCES}

1. W. Kauzmann, Chem. Rev., 43, 219 (1948).

2. M. C. Shen and A. Eisenberg, Prog. Solid State Chem., 3, 407 (1966).

3. A. J. Kovacs, J. Polym. Sci., 30, 131 (1958).

4. A. J. Kovacs, Fortschr. Hochpolymer. Forsch., 3, 394 (1963).

5. M. Goldstein and M. Nakonecznyj, Phys. Chem. Glasses, 6, 126 (1965).
6. S. Hozumi, Polym. J., 2, 756 (1971).

7. F. Buche, J. Chem. Phys., 36, 2940 (1962).

8. A. K. Doolittle, J. Appl. Phys., 22, 1471 (1951).

9. G. Adam and J. H. Gibbs, J. Chem. Phys., 43, 139 (1965).

10. S. Kästner, J. Polym. Sci., Part C, 16, 4121 (1968).

11. G. Williams, Trans. Faraday Soc., 59, 1397 (1963).

12. S. Saito and T. Nakajima, J. Appl. Polym. Sci., 2, 93 (1959).

13. A. J. Kovacs, R. A. Stratton, and J. D. Ferry, J. Phys. Chem., 67, 152 (1963).

14. T. G. Fox and P. J. Flory, J. Appl. Phys., 21, 581 (1950). 